\title{
Propiedad empresarial y calidad contable: evidencia en empresas españolas
}

\author{
Jaume Fernández Ramells \\ Máster en Contabilidad, Auditoría y Control de Gestión por la Universidad de Lleida. \\ *Correo de contacto: jfr8@alumnes.udl.cat \\ Recibido 9 de setiembre de 2019; Revisado 3 de noviembre de 2020; Aceptado 17 de noviembre 2020
}

\section{Resumen}

Estudios empíricos recientes miden la relación entre la propiedad empresarial y la calidad de la información contable. El presente trabajo analiza las prácticas de gestión o manipulación de los beneficios en el ámbito de estudio de empresas familiares y no familiares. Los resultados alcanzados muestran que, en comparación con las empresas no familiares, las empresas de naturaleza familiar divulgan unos resultados contables de mayor calidad, en términos de menores ajustes por devengo discrecionales. Además, se observa que las empresas con pérdidas tienen una relación positiva con la utilización de las prácticas de gestión de ganancias. Los resultados de este trabajo se enmarcan en la línea de investigación sobre las distintas tipologías de propiedad empresarial y la calidad contable. Los argumentos y justificaciones de nuestra investigación están vinculados al paradigma de riqueza socioemocional de la empresa familiar (SEW), cuyo objetivo es encontrar las razones tras las decisiones y el comportamiento de las empresas familiares, mediante la utilización de un análisis multidimensional.

\section{Clasificación JEL: G32, M41, M42}

Palabras Clave: riqueza socioemocional; propiedad empresarial; Empresa Familiar; problema de agencia.

\section{Introducción}

Las prácticas de gestión de ganancias ha sido un tema que ha captado la atención por parte de varios autores académicos. Los académicos como Osma et al. (2005), Ohlson (2014) o Klein (2002), han enfocado sus trabajos para hallar los motivos detrás de dichas prácticas y los distintos controles que existen para limitarlas. A raíz de la investigación realizada en las últimas décadas, se ha generado un debate entre la propiedad empresarial, es decir, entre empresas familiares y empresas no familiares y su relación con el uso de las prácticas de gestión de ganancias. Dicho debate surge con la aparición de dos efectos: el efecto de afianzamiento y el efecto de alineación. 
El efecto de afianzamiento o atrincheramiento, defendido por autores como Fan y Wong (2002) o Fama y Jensen (1985), establece que las empresas familiares ofrecen una calidad contable menor respecto a las empresas no familiares. El argumento principal de este efecto se basa en que las familias o accionistas controladores tienen incentivos para expropiar la riqueza de los accionistas minoristas. En cambio, el efecto de alienación, apoyado por autores como Anderson y Reeb (2003) o Demsetz y Lehn (1985), establece que las empresas familiares respecto a las empresas no familiares, tienen una relación positiva con la calidad contable, argumentando que existe una alineación de los intereses entre accionistas controladores y accionistas minoristas o entre gerentes y propietarios.

La teoría clásica de agencia entre gerentes y propietarios está relacionada con el debate que surge en el seno de las empresas familiares. Algunos autores como Bona et al. (2008) reflejan la aparición de un nuevo problema de agencia entre accionistas controladores, en el caso de empresas familiares, el problema de agencia surge entre las propias familias, y los accionistas minoritarios. Estos últimos son ajenos a la familia, por lo que están desligados de los intereses de trasmisión del control de la empresa a futuras generaciones. Les preocupa mucho más el beneficio inmediato que generar valor para la empresa pensando en el largo plazo.

Las firmas familiares tienen características de gobernanza únicas que se caracterizan por la dualidad de los objetivos económicos y no económicos que persiguen (Galve y Salas, 2011). Las firmas familiares se construyen mediante una estructura de gobierno dual que promueve la cohesión y la visión compartida dentro de la familia y en la que los miembros de la familia pueden desempeñar múltiples funciones en el negocio (Mustakallio et al., 2002). Por esto, el modelo de gobierno de empresas familiares es diferente al de otras empresas.

El debate que aparece en la literatura toma como referencia principalmente a empresas de mercados como el estadounidense (Ali et al., 2007), o el mercado alemán (Achleitner, 2014), entre otros. Bona et al. (2008) utiliza las empresas del mercado español para su análisis. El objetivo del trabajo es obtener unos resultados que permitan incorporarnos en el debate y conocer la relación entre las distintas tipologías de propiedad empresarial y la calidad de la información contable. La hipótesis inicial es que existe una relación positiva y significativa entre la empresa familiar y la calidad contable. Para contrastar la hipótesis, realizaremos una muestra de 93 empresas del mercado español con un período temporal entre 2011 y el 2017.

La metodología utilizada está en línea con la aplicada por otros autores como Achleitner et al. (2014) o Wang (2006) y difiere respecto a la metodología aplicada por Bona et al. (2008) en su trabajo debido a que incorpora la puntualidad asimétrica de las pérdidas. El modelo consiste en establecer la relación entre la empresa familiar y no familiar respecto a la calidad de contable, a través del uso de ajustes por devengo, y nos permite posicionarnos en el debate, en concreto, en el efecto alineación. Así, nuestros resultados establecen que la empresa familiar tiene una relación positiva y no significativa con la calidad contable en comparación a las empresas no familiares. Sin embargo, los resultados obtenidos tienen sus limitaciones, establecidas por una muestra reducida debido a la aplicación del filtro que promueve que solo tengamos en cuenta las empresas que cotizan, siendo un filtro muy utilizado por los académicos. Cabe añadir que nuestra diferencia entre las proporciones de empresas familiares y no familiares es 
mayor a la normalmente utilizada por otros autores como Achleitner et al. (2014) o Bona et al. (2008). Por tanto, si bien el modelo es robusto, los resultados se deberían considerar como aproximaciones en términos de extrapolar los resultados a todo el mercado español. Este aspecto ha sido criticado por varios autores por la utilización de muestras pequeñas en comparación a la magnitud de empresas que existen en el mercado, así como por el sesgo que existe en relación con la selección de filtros y períodos de la muestra. Además, no hay una uniformidad sobre la metodología aplicable. Por tanto, aún existen factores que se deben mejorar y la necesidad de teorías para justificar los comportamientos de las empresas respecto a la calidad contable.

El presente trabajo inicia con una visión general de la literatura sobre la propiedad empresarial y su relación con la calidad contable, analizando el debate y los distintos efectos. El siguiente apartado está destinado a la muestra y metodología utilizada en el trabajo. En el apartado cuarto se exponen los resultados de nuestro análisis para el mercado español. Finalmente, el trabajo finaliza resumiendo las principales conclusiones.

\section{Literatura e hipótesis}

Demsetz y Lehn (1985), Fan y Wong (2002), entre otros, establecen que la estructura de la propiedad empresarial afecta a la calidad de la información contable. A raíz de este hecho, en el presente trabajo intentamos comparar las empresas familiares y las empresas no familiares respecto a la calidad de la información contable.

La mayor parte de empresas del mundo son familiares (Wang, 2006). Las empresas familiares, a diferencia de las no familiares, representan una clase de accionistas que tienen una cartera poco diversificada, tienden a ser accionistas a largo plazo y tienen un mayor control sobre los puestos de gerenciales y directivos, siendo esencial el concepto de control transgeneracional y el de riqueza socioemocional (Socioemotional Wealth) para entender las diferencias respecto a las empresas no familiares, juntamente a sus motivaciones (Gómez-Mejía et al., 2007 y Zellweger et al., 2011).

La separación que surge entre la propiedad y control promueve el clásico problema de agencia citado por varios autores como Fama y Jensen (1983). El conflicto surge debido a que cuando hay una separación entre control y propiedad, existen ciertos incentivos para los gerentes basados en maximizar el beneficio personal a expensas de los accionistas o acreedores mediante el reporte de información financiera-contable. Wang (2006) establece que justamente son las ganancias contables las que se utilizan para intentar mitigar los conflictos de agencias al alinear los intereses de los directivos y gerentes con los intereses de los accionistas. En este caso, existiría una demanda de informes financieros-contables de calidad por parte de los accionistas con el fin de mejorar el control para que, a su vez, promoviera incentivos para que los directivos proporcionen estados financieros-contables de alta calidad. Así, las empresas familiares debido a su mejor capacidad de controlar directamente a los gerentes promueve que estos tengan un comportamiento menos oportunista (Ali et al., 2007 y Demsetz y Lehn, 1985).

La elevada protección legal al inversor genera unos mercados de capital más desarrollados y una menor concentración de la propiedad (Shleifer y Wolfenzon, 2002). Este aspecto, comentado en el punto anterior, es muy relevante debido a que, a diferencia de los países anglosajones en los que hay mayor nivel de protección legal a 
los inversores y existe una propiedad más difusa, los problemas de agencia que surgen son el clásico, es decir, entre propietarios y gerentes. No obstante, los países con mayores niveles de concentración de propiedad, como en Europa continental o algunos países de Asia, el problema clásico de agencia se reduce (Fama y Jensen, 1983), pero da lugar a conflictos de intereses entre los accionistas controladores, en una empresa familiar sería la propia familia, y los accionistas minoritarios (Shleifer y Vishny, 1997). En este nuevo contexto, los accionistas controladores pueden usar su poder e influencia para actividades destinadas a maximizar los beneficios privados que perjudiquen a los minoristas. Esto es así porque, como establecen Fan y Wong (2002), cuando un propietario controla efectivamente la empresa, también controla la producción de información contable y las políticas de los informes de la empresa.

Bona et al. (2008) establecen que existe otro aspecto para tener en cuenta en los países con elevada concentración de la propiedad y en los que predominan los conflictos de intereses entre los accionistas controladores y los minoritarios, que es la divergencia que se genera entre los derechos de voto y los derechos de los flujos de caja debido a que permite a los accionistas escapar de las consecuencias proporcionales de sus decisiones. Así, si los accionistas controladores tienen una elevada concentración de los votos, tienen incentivos para no alinear los intereses, es decir, que hay mayores incentivos para buscar los beneficios privados por parte de los accionistas controladores. En las empresas familiares, este hecho se podría acentuar más porque el beneficio se quedaría en la familia a diferencia de las empresas no familiares, donde se distribuiría entre los distintos accionistas. Como vemos, para reducir los conflictos de interés, es muy importante promover incentivos a los accionistas controladores para evitar que realicen prácticas que afecten negativamente a los intereses de los accionistas minoritarios (Ball et al., 2003).

En la literatura, surgen dos posiciones principales respecto a la estructura de propiedad y la calidad de la información contable. La mayoría de los autores se sitúan en una u otra posición, pues son excluyentes. Además, Wang (2006) establece que la propiedad familiar podría afectar a la oferta y demanda de la información contable a través de las dos posiciones: del efecto de afianzamiento o del efecto de alineación.

\subsection{El efecto de afianzamiento y las empresas familiares}

El efecto de afianzamiento se basa en el argumento de que la propiedad concentrada crea incentivos a los accionistas controladores o gerentes para expropiar la riqueza de otros accionistas. Por lo tanto, la idea es que los gerentes y directivos buscan la maximización de los beneficios privados a expensas de los propietarios o accionistas minoritarios (Fan y Wong, 2002; Morck et al., 1988; Shleifer y Vishny, 1997; Ball et al., 2003; Fama y Jensen, 1985 y 1983).

Este efecto representa el coste potencial de la propiedad familiar (Anderson y Reeb, 2003). El hecho de que la familia tenga fuerte control y poder en la toma de decisiones de la empresa puede afectar negativamente a su desempeño futuro. El fuerte control de la familia se puede manifestar en la selección del personal gerente y directivo de la empresa, generando que muchas veces sean los mismos miembros de la familia los que estén en los cargos directivos. Por tanto, se generan barreras por parte de la familia para que terceros no tomen el control de la empresa. 
Deangelo y Deangelo (2000) tratan la compañía que fue controlada por más de 100 años por la familia Chandler, la Time Mirror Company. Los autores constatan que la empresa experimentó un bajo rendimiento a partir de fines de los ochenta hasta mediados de los noventa. La razón establecida por los autores en relación con este desempeño empresarial fue el pago de dividendo especiales permitido por parte de la gerencia a los miembros de la familia Chandler con el objetivo de que la familia obtuviera dinero en efectivo a pesar de las bajas ganancias que tenía la empresa durante ese período. Además, estos dividendos fueron financiados especialmente a través de la venta de activos de la empresa y se garantizó que estos dividendos fueran a la familia a través de la política de dividendos, pero no se garantizó dividendos al resto de accionistas (accionistas minoritarios). Por tanto, es un ejemplo individual del efecto de afianzamiento debido a que hay una expropiación de la riqueza por parte de los accionistas controladores. Además, este ejemplo nos puede ofrecer una visión sobre el control que pueden llegar a tener las familias, las cuales pudieron modificar la política de dividendos de la empresa con el fin de maximizar sus beneficios privados a expensas del rendimiento operativo futuro de la empresa y de los accionistas minoritarios.

Chen, et al. (2008) en su trabajo nos establece que las empresas familiares tienden a una menor divulgación voluntaria de información en comparación a la empresa no familiar, afectando negativamente a la información contable. Estos hallazgos están en contraposición a los que establecen que las empresas familiares ofrecen una mejor calidad de la información defendidos por autores como (Ali et al., 2007). En concreto, los resultados de Chen, et al. (2008) establecen que las empresas familiares tienen menos probabilidades de emitir pronósticos a largo plazo de las ganancias $\mathrm{y}$, a corto plazo, divulgar buenas noticias sobre la empresa. No obstante, la empresa familiar tiene más probabilidad que las no familiares de emitir a corto plazo información sobre las malas noticias de ganancias. Las razones que encuentran los autores respecto a estos resultados es que las empresas familiares tienen menores incentivos de cumplir o superar las expectativas del mercado, puesto que sus accionistas, en este caso, la familia, tienen unos horizontes más a largo plazo, juntamente con mayor control y una menor asimetría de información entre propietarios y gerentes (este aspecto lo concretamos posteriormente). Además, las empresas familiares están más preocupadas por los costes que se derivan de problemas de litigios y que pueden afectar a la reputación de la familia. Por tanto, es más probable que a corto plazo emitan noticias no tan buenas sobre sus ganancias. En definitiva, Chen et al. (2008) nos ofrece unos resultados más afines al efecto de atrincheramiento, principalmente porque las empresas familiares tienden a ofrecer menor información de las ganancias, pudiendo afectar a su calidad.

En resumen, el efecto de atrincheramiento predice que las empresas familiares reportan ganancias de menor calidad que las empresas no familiares porque los miembros de la familia pueden tener mayores incentivos para administrar los beneficios y dedicarlos a autofinanciación.

\subsubsection{Efecto de afianzamiento y su impacto en la oferta}

Los resultados de Fan y Wong (2002) establecen que las propiedad familiar está asociada a un suministro de información sobre las ganancias de calidad más baja, generando un comportamiento más oportunista. Por tanto, es consistente con el efecto atrincheramiento. No obstante, el estudio de Fan y Wong (2002) se centra únicamente en los países del este asiático, impidiendo que sus resultados se puedan extrapolar hacia 
otros países con características de mercado distintas, como es el caso de Estados Unidos. Las características a las que hago más hincapié son que en Asia Oriental hay una mayor concentración de propiedad, unas leyes de protección a los inversores muy débiles y la información financiera es menos transparente que en los Estados Unidos.

Los países anglosajones son los principales países con mejores regulaciones y leyes de protección a los inversores, siendo determinante en el desarrollo de sus mercados (La Porta et al., 2000). Teniendo en cuenta Estados Unidos, si bien hemos comentado que no se puede extrapolar los resultados de Fan y Wong (2002) al caso del país anglosajón, parece ser que hay trabajos que establecen el efecto afianzamiento también en Estados Unidos. Francis et al. (2005) en su trabajo examinan la relación entre las ganancias y la información sobre los dividendos y las clases de acciones. Sus resultados establecen que las ganancias de las empresas con estructuras de capital dual son menos informativas que las ganancias de las empresas con estructuras de capital únicas. Sus hallazgos enfocados en Estados Unidos surgieren un efecto de afianzamiento de la propiedad familiar en la calidad de los ingresos porque las empresas de clase dual tienden a tener una mayor concentración de propiedad y se separa las decisiones de los accionistas controladores de los derechos de flujo de efectivo.

\subsubsection{El efecto de afianzamiento y su impacto en la demanda}

El efecto de afianzamiento predice que la propiedad familiar está asociada con una oferta de menor calidad de las ganancias. Por tanto, los usuarios de los estados financieros pueden exigir una mayor calidad de los ingresos en las empresas familiares. Las motivaciones por parte de la demanda son la protección de sus activos y sus intereses, pudiendo establecer términos de contratación que sean más sensibles a la calidad de las ganancias en las empresas familiares. Como consecuencia, el efecto de afianzamiento percibido de la propiedad familiar puede llevar a una mayor demanda de calidad de los ingresos por parte de los usuarios de los estados financieros y esto a su vez puede incentivar a las empresas familiares a reportar información sobre las ganancias de mayor calidad con el fin de mejorar los términos de contratación como, por ejemplo, préstamos con una reducción del coste de capital.

\subsection{Efecto de alineación y las empresas familiares}

El efecto de alineación se basa en la idea de que los intereses de los gerentes y los accionistas están alineados. En las empresas familiares sería una alineación entre los intereses de la familia y los otros accionistas. Por tanto, el efecto sugiere que es menos probable que las familias (accionistas controladores) o los gerentes expropien riquezas de los otros accionistas a través de la administración de ganancias (Achleitner et al., 2014; Wang, 2006; Anderson y Reeb, 2003; Bona et al., 2008; Demsetz y Lehn, 1985; Maury, 2006).

Demsetz y Lehn (1985) encuentran la evidencia basada en que los accionistas controladores tienen incentivos para reducir los problemas de agencia y maximizar el valor de la empresa debido a que la riqueza familiar está estrechamente vinculada al bienestar de la empresa. Por lo tanto, las familias tienen fuertes incentivos para monitorear a los gerentes, mejorando su supervisión a largo plazo y avanzar más en la curva de aprendizaje. Además, el monitoreo o supervisión permite minimizar el problema inherente a los accionistas minoritarios, es decir, reducir el problema de agencia. 
Varios autores como Achleitner et al. (2014), Anderson y Reeb (2003) o James (1999) establecen que un aspecto clave que promueve la alineación de intereses es que las empresas familiares tienen horizontes de inversión a más largo plazo que la empresas no familiares. James (1999) en su trabajo pone en relevancia que los extensos horizontes de las familias pueden proveer de los incentivos necesarios para tomar decisiones con el fin de invertir según las reglas del mercado, al mismo tiempo que reducen los costes de agencia. A parte de esto, el autor hace referencia a unos valores imprescindibles que caracterizan a las empresas familiares como son los lazos familiares, la lealtad, la protección y la estabilidad familiar debido a que estos valores evidentemente juegan un papel trascendental en la toma eficiente de decisiones sobre las inversiones y su efecto a largo plazo.

Los horizontes en la empresas familiares, según el trabajo de James (1999), se pueden extender por dos vías principalmente: primero a través de las relaciones interpersonales entre los miembros de la familia que forjan lazos entre los miembros de la familia y las futuras generaciones; y segundo a través de la transferencia de bienes no pecuniarios entre padres e hijos/hijas que ofrecen seguridad y protección. En referencia a la primera, el autor destaca dos valores relevantes como son los lazos familiares y la lealtad. Estos valores, inherentes a una empresa familiar, promueven acciones que permiten beneficiar a los otros miembros de la empresa, es decir, las empresas familiares pueden sacrificar los beneficios actuales con el fin de obtener beneficio a largo plazo para todos los miembros del a familia. En relación con la segunda vía, la protección familiar es un valor muy relevante pues los propietarios reconocen la conexión entre la viabilidad empresarial y su habilidad para proteger a los miembros de la familia de los futuros riesgos. Por tanto, la protección familiar puede generar eficiencia en la toma de decisiones con una perspectiva a largo plazo.

La mayoría de las empresas familiares no sobreviven a muchas generaciones. Así, James (1999) establece que en Estados Unidos un 70\% de las firmas familiares no sobreviven a la segunda generación familiar. El autor atribuye tal fracaso a la inestabilidad de la familia, es decir, si hay inestabilidad en la familia, las empresas familiares no podrían mantener valores importantes como la lealtad intergeneracional. Otro aspecto relevante es la capacidad de los hijos, es decir, en ocasiones puede aparecer la situación de que los padres no quieran transferir la empresa a sus hijos, limitando la toma de inversiones óptimas, afectando a la extensión de los horizontes familiares.

En definitiva, el efecto de alineación consiste en que la propiedad familiar crea incentivos para que los miembros de la familia maximicen la riqueza de todos los accionistas. Por tanto, la extensión del horizonte de inversión a largo plazo, una mayor participación en la empresa, la existencia de un conjunto de valores y los incentivos para preservar la reputación de la familia, limitan la actuación oportunista de las familias en relación con la gestión de ganancias contables.

\subsubsection{Efecto de alineación y su impacto en la oferta de información financiera}

El efecto de alineación en la empresa familiar parte del supuesto de que la propiedad familiar está asociada a un fuerte gobierno corporativo debido a la aplicación de un conjunto de valores. El hecho de tener un gobierno corporativo fuerte mitiga el oportunismo de los gerentes en la manipulación de las ganancias reportadas. Las familias tienen incentivos para informar de ganancias de mayor calidad mediante un uso 
de la contabilidad que evite daños potenciales a la reputación familiar y al desarrollo a largo plazo de la empresa. Por tanto, las empresas familiares pueden informar de beneficios de mayor calidad que las empresas no familiares y por tanto, presentar beneficios no manipulados.

En las empresas familiares, los miembros de la familia tienden a poseer elevados porcentajes de participación en el capital-propiedad. Warfieid et al. (1995) establecen una relación positiva entre la propiedad gerencial y la calidad de la información contable. Como la propiedad es mayoritariamente de la familia, los autores sugieren un efecto de alineación entre propiedad y gerencia, por ello, se espera que las empresas familiares reporten ganancias de mayor calidad.

\subsubsection{Efecto de alineación y su impacto en la demanda de información financiera}

Desde un punto de vista de los accionistas o acreedores, si existe un gobierno corporativo fuerte y una alineación de intereses, puede llevar una menor demanda de informes contables de calidad. Los acreedores si creen que la propiedad familiar está asociada con un gobierno corporativo más fuerte, los términos de contratación de la deuda son menos sensibles a la calidad de las ganancias contables porque asumen que los estados financieros-contables se elaboran correctamente.

Por tanto, el efecto de alineación implica que la propiedad familiar reduce la demanda de informes financieros-contables de calidad. Respecto a esta relación es importante tener en cuenta a autores como Ball y Shivakumar (2005) y Ball et al. (2003) debido que establecen que las ganancias son de menor calidad, en el caso de que los incentivos para reportar la información sean bajos. Por lo tanto, los incentivos juegan el principal papel para mejorar o empeorar la calidad de las ganancias. En su trabajo Ball et al. (2003) tienen como resultados que las empresas familiares reportan ganancias de menor calidad porque sus incentivos para reportar ganancias de alta calidad son más bajos que en las empresas no familiares.

En el presente trabajo, analizaremos el impacto de la estructura empresarial en la calidad de la información contable. Los datos que utilizaremos son de las empresas españolas, donde más del 80\% de las empresas son familiares. Bona et al. (2008) realiza su trabajo utilizando las empresas españolas como muestra para su análisis. En su trabajo establece que España es un país con bajas leyes de protección a los inversores, afectando al desarrollo de sus mercados financieros y promoviendo una mayor concentración de la propiedad. No obstante, si bien estas afirmaciones pueden ofrecer una idea a priori de que en España puede que haya un efecto de afianzamiento, es decir, una relación negativa entre empresa familiar y la calidad de las ganancias, los hallazgos de Bona et al. (2008) establecen que la relación es positiva. Por tanto, a partir de estas afirmaciones, partimos de una hipótesis basada en el efecto de alineación. La hipótesis inicial es:

Hp: La empresa familiar tiene un efecto positivo y significativo sobre la calidad contable de las ganancias.

\section{Datos y metodología}

En este estudio utilizaremos como muestra las empresas no financieras españolas con un período que comprende desde 2011 hasta 2017. No obstante, debido a la enorme 
cantidad de estas, decidimos tener en cuenta las empresas que cotizan por motivos de mayor facilidad y transparencia de los datos publicados sobre su situación contable y financiera. Este filtro es utilizado por la mayoría de los autores mencionados anteriormente. En este caso, podemos destacar Achleitner et al. (2014) para el caso alemán, Ali et al. (2007) y Anderson y Reeb (2003) para el caso de Estados Unidos, y Bona et al. (2008) selecciona empresas del mercado bursátil español.

La aplicación del primer filtro, de exigir que sean compañías cotizadas, reduce a una muestra de 153 empresas, compuesta por 105 empresas familiares y 48 empresas no familiares. Los datos, tanto de las empresas como de las variables utilizadas para el análisis posterior, proceden de la base de datos SABI. Posteriormente se han eliminado 43 empresas familiares y 17 empresas no familiares por motivos de insuficiencia de datos durante el período 2011-2017 en determinadas variables utilizadas en la metodología. Por tanto, la muestra final está compuesta por un total de 93 empresas entre 2011 y 2017, 62 empresas familiares y 31 empresas no familiares. La muestra total final tiene un número de empresas similar a la muestra de Bona et al. (2008) para el caso español.

Para realizar un breve análisis de las empresas, en la tabla 1 se muestran en promedio, algunas de las variables utilizadas en la metodología en el 2017. Los datos de la tabla muestran que las empresas familiares presentan una mejor rentabilidad de los activos en promedio conjunto que las empresas no familiares. No obstante, las empresas no familiares presentan un crecimiento de la cifra de negocios superior y un nivel de apalancamiento menor.

La tabla 2 es referente al 2016 con unos resultados conjuntos similares entre empresas familiares y no familiares respecto al 2017. Si tenemos en cuenta la evolución y partimos de que se trata de promedios, la rentabilidad de los activos se ha reducido en general, mientras que el crecimiento de la cifra de negocios ha incrementado, juntamente con el nivel de apalancamiento. Las empresas no familiares tienen un mayor crecimiento de la cifra de negocios respecto a las empresas familiares. En referencia al apalancamiento, la mayor variación es en la empresa no familiar, siendo explicado en parte por la variación de la cifra de negocios. En referencia a los auditores, tanto empresas familiares como empresas no familiares son auditadas en un porcentaje superior al $80 \%$ por las Big Four ${ }^{1}$, siendo sensiblemente mayor en el caso de las empresas no familiares.

Tabla 1. Variables muestra en el año 2017

\begin{tabular}{|l|c|c|c|c|}
\hline & ROA & $\begin{array}{c}\text { Crecimiento de la } \\
\text { cifra de negocios }\end{array}$ & Apalancamiento & Auditores \\
\hline Empresa Familiar & 13,22 & 56,80 & 135,50 & 0,82 \\
\hline Empresa no Familiar & 1,39 & 392,01 & 64,15 & 0,87 \\
\hline
\end{tabular}

Fuente: Elaboración propia a partir de los datos de la muestra.

\footnotetext{
${ }^{1}$ Las Big Four son las firmas más importantes del mundo en el sector de la consultoría y auditoría. Las firmas son Deloitte, PricewaterwhouseCooper (PWC), KPMG y Ernst \& Young.
} 
Tabla 2. Variables muestra en el año 2016

\begin{tabular}{|l|c|c|c|c|}
\hline & ROA & $\begin{array}{c}\text { Crecimiento de la } \\
\text { cifra de negocios }\end{array}$ & Apalancamiento & Auditores \\
\hline Empresa Familiar & 17,39 & 14,63 & 122,94 & 0,82 \\
\hline Empresa no Familiar & 3,20 & 38,18 & 47,18 & 0,87 \\
\hline
\end{tabular}

Fuente: Elaboración propia a partir de los datos de la muestra.

Con el objetivo de comprobar nuestra hipótesis de trabajo, realizaremos un modelo basado en los ajustes por devengo discrecionales. El modelo inicialmente fue desarrollado por Dechow y Dichev (2002) y posteriormente se modificó por Ball y Shivakumar (2005). Esta metodología ha sido realizada por otros autores como Wang (2006) y Achleitner et al. (2014). Es importante remarcar que esta metodología es diferente al modelo convencional de investigaciones previas con el carácter lineal de los ajustes por devengo discrecionales, utilizado por otros autores como Jones (1991), el cual posteriormente fue modificado por Dechow, Sloan y Sweeney (1995) y fue utilizado por otros autores como Bona et al. (2008) en el caso de España. La diferencia de este modelo, como establece Achleitner et al. (2014), es que el modelo incorpora la puntualidad asimétrica de las pérdidas.

La hipótesis de nuestro trabajo es:

Hp: La empresa familiar tiene un efecto positivo y significativo sobre la calidad contable de las ganancias.

Para estimar los ajustes por devengo, utilizaremos la siguiente ecuación y realizaremos una regresión de mínimos cuadrados ordinarios (MCO):

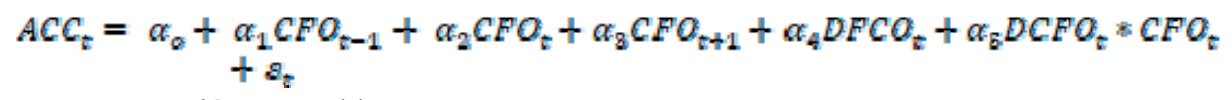

En esta ecuación se establece:

- $A C C_{\text {t }}$ son los 'accruals' totales en el año $t$ entendidos como la diferencia entre los ingresos netos antes de partidas extraordinarias y los flujos de caja operativos. Posteriormente, tenemos que dividir la operación por la media de los activos en el año $t$.

- $\mathrm{CFO}_{\mathrm{r}}$ son los flujos de caja operativos el año $t$ divididos por la media de los activos en el año $t$.

- $\quad \mathrm{CPO}_{t+1}$ son los flujos de caja operativos el año $t+1$ divididos por la media de los activos en el año $t+1$.

- $\mathrm{CFO}_{\mathrm{r}-1}$ son los flujos de caja operativos el año $t-1$ divididos por la media de los activos en el año $t-1$.

- $\quad \mathrm{DFCO}_{\mathrm{\tau}}$ es un indicador que es igual a uno si los flujos de caja operativos en $t$ son negativos, cero en el caso contrario.

- $\quad D C F O_{\tau} * C F O_{\tau}$ es un proxy de pérdidas económicas.

Una vez realizada la regresión, para tener un proxy de la gestión de ganancias, utilizaremos el valor de los residuos, creando una nueva variable $A B S A C C_{t}$. Siguiendo a Achleitner et al. (2014), realizaremos otra regresión utilizando como variable exógena una variable dummy que permita discernir entre empresa familiar y no familiar, 
juntamente con otras variables control. Para ello, se ha utilizado el indicador de independencia de la base de datos SABI que permite diferenciar las empresas en función del nivel del porcentaje de propiedad empresarial que tienen los accionistas. Utilizaremos la siguiente ecuación para verificar la hipótesis:

\section{$A B S A C C_{t}=\alpha_{0}+\beta_{1}$ FAM $+\beta_{2}{ }_{2} O A_{t}+\beta_{g} A P A L A N C A M I E N T O_{t}$ $+\beta_{4}$ CRECIMIENTO $0_{\tau}+\beta_{8}$ PERDIDAS $_{\tau}+\beta_{6} A U D_{\tau}+s_{\tau}$}

- $A B S A C C_{8}$ es el proxy de la gestión de ganancias en $t$.

- $F^{A} M_{0}$ es una variable dummy que es cero si la empresa no es familiar, uno en caso contrario. Se utiliza el indicador de independencia para la distinción entre la propiedad de empresas.

- $R O A_{t}$ es la rentabilidad de los activos en $t$.

- APALANCAMTENTO es $_{\text {el }}$ apalancamiento en el año $t$, entendido como la división entre el pasivo total entre los fondos propios.

- CRECIMTENTO es el porcentaje de crecimiento del importe de la cifra de negocios en $t$.

- PEFDIDAS: es una variable dummy en la que cero es que la empresa tiene perdidas en el año $t$, uno en caso contrario.

- $A U D_{0}$ es una variable dummy, siendo uno si la empresa es auditada por una Big Four en $t$, cero en caso contrario. Esta variable control es introducida por Achleitner et al. (2014) en su trabajo.

La primera regresión, procedente de la aplicación de la primera fórmula, tiene como objetivo principal obtener un proxy de la gestión de ganancias, a través de los residuos del modelo. Para analizar la robustez del modelo, hemos realizado distintos contrastes y pruebas (véase la tabla 3). Así, el modelo no presenta problemas de heterocedasticidad y no existe multicolinealidad. En la tabla 4 se muestra el modelo. Una limitación que puede afectar al modelo final es que, si bien es verdad que el modelo obtenido de la primera ecuación es robusto, el coeficiente de determinación es bajo, es decir, que el modelo estimado explica poco de la variabilidad de la endógena. Relacionado con esta limitación del trabajo, en los trabajos de otros autores como Achleitner et al. (2014) no establecen una importancia remarcable sobre este aspecto en relación con el modelo, puesto que su principal función es obtener los residuos del mismo.

Tabla 3. Pruebas para comprobar la veracidad de los modelos

\begin{tabular}{|l|c|}
\hline Test & ACC \\
\hline Contraste de Heterocedasticidad de White & 19,79 \\
\hline Multicolinealidad & $\begin{array}{l}\text { Los valores (VIF) } \\
\text { inferiores a 10 en } \\
\text { todas las variables }\end{array}$ \\
\hline
\end{tabular}

Nota: El contraste de White tiene como hipótesis nula que no hay heterocedasticidad, por tanto, hay homocedasticidad y la hipótesis alternativa es que hay heterocedasticidad. El contraste de multicolinealidad sirve para verificar que no existe una fuerte dependencia lineal entre las variables exógenas. Los niveles de rechazo de la hipótesis nula: *significación $10 \%$, **significación $5 \%$, ***significación $1 \%$. 
Tabla 4. Modelo para obtener ABS ACC.

\begin{tabular}{|lc|}
\hline & Modelo 1 \\
\hline Variable dependiente & ACC \\
\hline CF & 0,1885 \\
& $(0,5157)$ \\
CF-1 & $-0,4049$ \\
& $(0,3541)$ \\
CF+1 & $-0,3459$ \\
& $(0,4882)$ \\
DFCO & 0,0121 \\
& $(0,0876)$ \\
DFCO*CF & $-0,1137$ \\
& $(0,6427)$ \\
\hline Constante & 0,0533 \\
& $(0,0592)$ \\
\hline
\end{tabular}

Estimaciones realizadas con Mínimos Cuadrados Ordinarios (MCO). En paréntesis: *significación 10\%, $* *$ significación $5 \%, * * *$ significación $1 \%$.

Una vez obtenido el proxy de gestión de ganancias con la utilización de los residuos del modelo anterior. Establecemos la regresión correspondiente a la segunda ecuación, obteniendo el modelo necesario para establecer nuestros resultados y conclusiones. El modelo inicial obtenido presentó problemas de heterocedasticidad. Por tanto, se ha modificado el modelo para corregir dicho problema. El modelo final corresponde a la tabla 5. En el modelo se han realizado las correspondientes pruebas y test para analizar la comprobar su robustez. Así, como se muestra en la tabla 6, el modelo no presenta problemas en la distribución de los residuos ni problemas de multicolinealidad. 
Tabla 5. Relación entre los accruals y la propiedad empresarial.

\begin{tabular}{|c|c|c|c|}
\hline \multirow{2}{*}{\begin{tabular}{|l} 
Variable dependiente \\
FAM
\end{tabular}} & \multirow{2}{*}{$\begin{array}{c}\text { Signo esperado } \\
-\end{array}$} & \multicolumn{2}{|c|}{$\begin{array}{l}\text { Modelo } 2 \\
\text { ABS ACC }\end{array}$} \\
\hline & & $\begin{array}{l}-0,0089 \\
(0,0200)\end{array}$ & \\
\hline ROA & & $\begin{array}{c}0,0015 \\
(0,0153)\end{array}$ & $* * *$ \\
\hline Apalancamiento & & $\begin{array}{c}0,0150 \\
(0,0121)\end{array}$ & \\
\hline Crecimiento & & $\begin{array}{l}-0,0127 \\
(0,0168)\end{array}$ & \\
\hline Perdidas & & $\begin{array}{c}0,1110 \\
(0,0234)\end{array}$ & $* * *$ \\
\hline Auditores & & $\begin{array}{c}0,0245 \\
(0,0261)\end{array}$ & \\
\hline Constante & & $\begin{array}{l}-0,1538 \\
(0,0325)\end{array}$ & $* * *$ \\
\hline $\mathrm{R}^{2}$ & & 0,62 & \\
\hline Observaciones & & 465 & \\
\hline
\end{tabular}

Estimaciones realizadas con Mínimos Cuadrados Ordinarios (MCO). En paréntesis: *significación 10\%, $* *$ significación $5 \%, * * *$ significación $1 \%$.

Tabla 6. Pruebas para comprobar la veracidad del modelo.

\begin{tabular}{|l|c|}
\hline Test & ABS ACC \\
\hline Multicolinealidad & $\begin{array}{l}\text { Los valores (VIF) } \\
\text { inferiores a 10 en } \\
\text { todas las variables }\end{array}$ \\
\hline Contraste de normalidad de los residuos & 0,379 \\
\hline
\end{tabular}

El contraste de multicolinealidad sirve para verificar que no existe una fuerte dependencia lineal entre las variables exógenas. El contraste de normalidad en los residuos tiene como hipótesis nula que el error se distribuye normalmente. El modelo no presenta problemas en la distribución de los residuos. 


\section{Resultados}

En el modelo establecido en la tabla 5, tiene un coeficiente de determinación mayor, explicando un $62 \%$ de la variabilidad de la variable endógena. El principal resultado del modelo es el no rechazo a la hipótesis inicial debido a que la existe una relación positiva entre la empresa familiar y el menor uso de ajustes por devengo discrecionales. No obstante, la relación no es significativa.

Los resultados del presente trabajo están relacionados con la existencia de una alineación entre los intereses de los gerentes y accionistas o entre los accionistas controladores y los minoristas, reduciendo el problema de agencia. Además, si bien en el mercado español existe una baja protección a los accionistas minoritarios (Bona et al., 2008) y, por tanto, afectaría negativamente al desarrollo de los mercados (La Porta, et al., 2000; Shleifer y Wolfenzon, 2002 y Sánchez y Fuentes, 2008), generando una idea a priori de la existencia de un efecto de afianzamiento, nuestros resultados ofrecen una relación distinta a la esperada. Así, nuestros resultados son similares a los encontrados por Bona et al. (2008) para el caso español. No obstante, nuestros resultados, a pesar de mostrar la relación positiva, la relación no es significativa. Los motivos pueden estar relacionados, entre otros, por un nivel bajo de observaciones, en comparación a otros autores como Wang (2006) o Achleitner et al. (2014); por la metodología empleada; o por motivos procedentes de la diferencia entre las proporciones de empresa familiar y no familiar utilizadas en la muestra respecto a otros trabajos como el Bona et al. (2008), cuya diferencia entre empresa familiar y empresa no familiar era menor.

El resultado nos incluye dentro del efecto de alineación, juntamente con otros autores como Achleitner et al. (2014), Wang (2006), Anderson y Reeb (2003), Demsetz y Lehn (1985) y Maury (2006). Las razones con las que podemos justificar la relación positiva entre empresas familiar y la calidad contable son la mayor monitorización por parte de los accionistas controladores (la familia) a los gerentes que pueden ser los propios miembros de la familia, promoviendo una supervisión a largo plazo y minimizando el problema propio de los accionistas minoritarios, es decir, reduciendo el problema de agencia. Las empresas familiares tienen unos horizontes de inversión a largo plazo (Anderson y Reeb, 2003 y James, 1999), creando incentivos para tomar decisiones que reduzcan los problemas de agencias y busquen generar la mejora y supervivencia empresarial a largo plazo debido a que, en muchas ocasiones, la situación empresarial está ligada al bienestar de la familia controladora.

La relación puede ser justificada desde el paradigma de la riqueza socioemocional. Así, si bien establece que, en las empresas familiares, pueden existir ciertos comportamientos oportunistas, los cuales podrían suponer un elevado riesgo para la empresa, cuyo objetivo sea reducir las amenazas en la riqueza socioemocional (Berrone et al., 2012), el paradigma ofrece un conjunto de razones que justifican los resultados encontrados en el presente trabajo. Un aspecto remarcable es el control familiar sobre las decisiones estratégicas, que normalmente está ligado con los horizontes a largo plazo y el control transgeneracional, generando una motivación por parte de los accionistas controladores en relación con las prácticas contables de calidad con el fin de preservar la continuidad de la empresa (Gómez-Mejía et al., 2007). Además, la familia se identifica con la empresa, siendo la reputación familiar otro aspecto que puede justificar la tendencia a una mejora contable respecto a las empresas no familiares (Berrone et al., 2010). Otro factor por destacar es el componente emocional que, a pesar de ser un 
ámbito dentro del paradigma socioemocional aún en desarrollo, puede ofrecer una justificación a nuestros resultados debido al sentimiento que se tiende a crear de compromiso, estabilidad y lealtad en las empresas familiares.

En conclusión, el primer resultado y más relevante es que las empresas familiares tienen una relación positiva y no significativa respecto a la calidad contable, rechazando la línea académica basada en que los accionistas controladores o gerentes, en función del problema de agencia, maximizan sus beneficios privados a expensas de los otros accionistas. Por tanto, nuestros resultados se encuentran en la línea del efecto de alineación.

En relación con las variables control, podemos destacar que existen dos variables, la rentabilidad de los activos y pérdidas, cuyas relaciones con la variable dependiente son positivas y significativas. Estas relaciones las podemos encontrar en otros autores. Así, la relación positiva con la rentabilidad de los activos también se establece en el artículo de Bona et al. (2008). La relación se puede explicar cómo que la utilización de ajustes discrecionales tiende a incrementar la rentabilidad de los activos, ya sea a través del incremento artificial del beneficio o de la reducción de activos, por ejemplo, mediante ventas de activos o por otros motivos que impliquen estimaciones más subjetivas como la amortización o los deterioros. En relación con la variable pérdidas, si bien la relación es contraria a la establecida por (Achleitner et al., 2014), es coherente con la establecida por Bona et al. (2008) y Wang (2006), estableciendo que hay una relación positiva entre las empresas que presentan pérdidas y la utilización de ajustes discrecionales. Uno de los posibles motivos para reducir el beneficio y entrar en pérdidas es minorar los costes fiscales.

Las últimas variables apalancamiento, crecimiento y auditores son utilizadas por otros autores como variables control $\mathrm{y}$, en nuestro caso, ninguna establece una relación significativa. Bona et al. (2008) también establece una relación positiva entre la utilización de ajustes discrecionales y el apalancamiento. Esta relación se puede justificar por la motivación contractual. Así, en empresas con grandes deudas y delante de la dificultad de cumplir los períodos establecidos, los gerentes o familias tienen incentivos para realizar ajustes discrecionales, transfiriendo beneficios futuros al momento actual si el ratio de apalancamiento es elevado. La variable crecimiento establece una relación negativa, siendo una relación distinta a la establecida por otros autores como Achleitner et al. (2014) o Wang (2006). La relación establecida por los autores se justifica con que la utilización de los ajustes discrecionales tiene una relación positiva con el crecimiento de las ventas. No obstante, nuestro resultado es la relación opuesta, pudiéndose justificar en relación con el resultado obtenido con la variable perdidas, es decir, que las empresas que tienen pérdidas tienden a utilizar ajustes discrecionales y, por tanto, esto puede suceder reduciendo artificialmente el importe de la cifra de negocios, afectando negativamente a su crecimiento. Finalmente, el resultado de la variable auditoria establece una relación opuesta a la utilizada por Achleitner et al. (2014) en su trabajo. Así, nuestro resultado establece que las grandes firmas de auditoría tienen una relación positiva con la utilización de ajustes discrecionales. La auditoría es, sin duda, un factor de control a la manipulación y, por tanto, la relación debería ser teóricamente negativa. No obstante, los clientes de las grandes firmas de auditoría son grandes empresas multinacionales con unos circuitos de contabilización de ingresos muy complejos. Además, deberíamos tener en cuenta la independencia y la importancia relativa que se incorpora en toda la fase de ejecución del trabajo de auditoría, pudiendo 
afectar a que existan ajustes discrecionales sin que los auditores los detecten. Las últimas tres variables no son significativas y son variables control, por tanto, a pesar de establecer una justificación, los resultados no son definitivos.

\section{Conclusiones y discusión de resultados}

En el presente trabajo se ha analizado prácticas de gestión de ganancias, así como sus motivaciones y controles. El objetivo del trabajo es conocer la relación entre las estructuras de propiedad familiar y la calidad contable. Así, a través de un análisis de la literatura, hemos obtenido un marco teórico basado en dos posiciones. Por un lado, existen autores, entre los cuales podemos destacar a Fan y Wong (2002) o Shleifer y Vishny (1997), que apoyan con sus trabajos al efecto de afianzamiento, cuyo argumento está basado en el que los accionistas controladores tienen incentivos para expropiar la riqueza de los accionistas minoritarios, aunque en función del problema de agencia que tratemos, el conflicto puede ser entre gerentes y propietarios. El argumento se puede traducir en que las empresas familiares, donde la familia tiene un elevado control, pueden tener incentivos para utilizar prácticas de gestión de ganancias respecto a las empresas no familiares. Por otro lado, autores como Bona et al. (2008), Wang (2006) o Maury (2006), establecen unos resultados que se incluyen en el efecto de alienación, cuya argumentación está basada en la alineación de intereses entre los gerentes y accionistas. La congruencia de intereses provocaría unos bajos incentivos para que los accionistas controladores expropiasen las riquezas a los accionistas minoritarios. Así, el efecto de alineación establece que la propiedad familiar tiene incentivos para que los miembros de la familia (gerentes, accionistas controladores), maximicen la riqueza de todos los accionistas y se limite la actuación oportunista. Por tanto, el trabajo se encuentra enmarcado dentro del debate vigente en la literatura académica.

La hipótesis del trabajo es que existe una relación positiva entre la empresa familiar y la calidad contable. Así, nuestros resultados obtenidos para el caso español no rechazan la hipótesis establecida y, por tanto, establecen que la empresa familiar tiene una relación positiva con la calidad contable respecto a las empresas no familiares, aunque dicha relación no es significativa. El resultado obtenido nos incluye dentro del efecto alienación. Además, el modelo establece que una relación positiva entre las empresas con pérdidas y la utilización de ajustes por devengo discrecionales, siendo la misma relación, con el mismo nivel de significación, con las empresas que buscan incrementar la rentabilidad de sus activos. Los resultados obtenidos están acorde con los establecidos por Bona et al. (2008), cuyo trabajo también se realizó sobre el mercado español.

Sin embargo, nuestro trabajo tiene ciertas limitaciones que pueden afectar en cierto modo a los resultados obtenidos. En la muestra se ha establecido un filtro utilizado por la mayoría de los autores dejando una muestra total de 93 empresas. Por tanto, para obtener unos resultados más fiables, se debería utilizar una muestra con un mayor número de observaciones y con una mejor proporción entre empresas familiares y empresas no familiares. Una segunda limitación está relacionada con la metodología empleada debido a que existen diferencias metodológicas entre autores, pudiendo generar discordancia entre los resultados obtenidos.

Un camino hacia la explicación de los distintos efectos establecidos en la literatura académica es el paradigma de la riqueza socioemocional. El paradigma, aún en desarrollo, puede ofrecer una vía para entender las razones detrás del comportamiento 
de las empresas familiares, mediante un análisis multidimensional. SEW no descarta ninguno de los efectos, pues considera que, en las empresas familiares, puede haber comportamientos oportunistas, al mismo tiempo que establece que, por determinados factores como el control transgeneracional, la lealtad o la identificación familiar, las empresas familiares tienen incentivos para mejorar la situación de la empresa y, por tanto, una menor probabilidad de utilizar prácticas de gestión de las ganancias.

El presente trabajo se incorpora en el debate académico vigente entre propiedad empresarial y calidad contable. Los resultados apuntan hacia un efecto de alineación, es decir, que las empresas familiares utilizan menos ajustes por devengo discrecionales que las empresas no familiares. Las limitaciones establecidas en la literatura, así como las diferencias en la metodología empleada y la aplicación del nuevo enfoque basado en el paradigma de la riqueza socioemocional son líneas de investigación que se deben desarrollar en el futuro bajo el fin de conocer el comportamiento de las empresas según su estructura de propiedad y su efecto en la utilización de las prácticas de earning management.

\section{Referencias}

Achleitner, A.; Günther, N.; Kaserer, C. y Gianfranco, S. (2014). "Real Earnings Management and Accrual-based Earnings Management in Family Firms". European Accounting Review, 23(3), 431-462.

Ali, A.; Chen, T. y Radhakrishnan, S. (2007). "Corporate disclosures by family firms". Journal of Accounting and Economics, 44, 238-286.

Anderson, R. y Reeb, D. M. (2003). "Founding-Family Ownership and Firm Performance: Evidence from the S\&P 500". The Journal of Finance, LVIII(3), 13011328.

Ball, R.; Kothari, S. P.; Robin, A.; Biddle, G.; Carsberg, S. B.; Collins, D.; ... Zimmerman, J. (2000). "The effect of international institutional factors on properties of accounting earnings". Journal of Accounting and Economics, 29, 1-51.

Ball, R.; Robin, A. y Shuang, J. (2003). "Incentives versus standards: properties of accounting income in four East Asian countries". Journal of Accounting and Economics, 36, 235-270.

Ball, R. y Shivakumar, L. (2005). "Earnings quality in UK private firms: comparative loss recognition timeliness". Journal of Accounting and Economics, 39, 83-128.

Berrone, P.; Cruz, C.; Gomez-mejia, L. y Larraza-kintana, M. (2010). "Socioemotional Wealth and Corporate Responses to Institutional Pressures: Do Family-Controlled Firms Pollute Less ?" Administrative Science Quaterly, 55(1), 82-113.

Berrone, P.; Cruz, C. y Gomez-Mejia, L. R. (2012). "Socioemotional Wealth in Family Firms". Family Business Review, 25(3), 258-279.

Bona, C.; Pérez, J. y Santana, D. J. (2008). "Family control and earnings quality". Revista de Contabilidad, 10(1), 11-34.

Chen, S.; Chen, X. y Cheng, Q. (2008). "Do Family Firms Provide More or Less Voluntary Disclosure?" Journal of Accouting Research, 46(3), 499-536.

Deangelo, H. y Deangelo, L. (2000). "Controlling stockholders and the disciplinary role of corporate payout policy: a study of the Times Mirror Company". Journal of 
Financial Economics, 56, 153-207.

Dechow, P. y Dichev, I. (2002). "The quality of accrual and earnings: The role of accrual estimation errors". The Accounting Review, 77, 35-59.

Dechow, P.; Sloan, R. y Sweeney, A. (1995). "Detecting Earnings Management". The Accounting Review, 70(2), 193-225.

Demsetz, H. y Lehn, K. (1985). "The Structure of Corporate Ownership: Causes and Consequences". Journal of Political Economy, 93(6), 1155-1177.

Fama, E. y Jensen, M. (1983). "Separation of Ownership and Control". Journal of Law Economics, 26(2), 301-326.

Fama, E. y Jensen, M. (1985). "Organizational forms and investment decisions". Journal of Financial Economics, 14, 101-119.

Fan, J. P. H. y Wong, T. J. (2002). "Corporate ownership structure and the informativeness of accounting earnings in East Asia". Journal of Accounting and Economics, 33, 401-425.

Francis, J.; Schipper, K.; Vincent, L.; Beaver, B.; Blacconiere, W.; Butler, A.; ... Watts, R. (2005). "Earnings and dividend informativeness when cash flow rights are separated from voting rights". Journal of Accounting and Economics, 39, 329-360.

Galve-Górriz, C. y Salas-Fumás, V. (2011) "Family ownership and firm performance: the net effect of productive efficiency and growth constraints". Innovar, 21(40), pp. $155-170$

Gómez-mejía, L.; Takács, K.; Núñez-Nickel, M. y Moyana-fuentes, J. (2007). "Socioemotional Wealth and Business Risks in Family-Controlled Firms: Evidence from Spanish Olive Oil Mills". Administrative Science Quaterly, 52(1), 106-137.

James, H. S. (1999). "Owner as Manager, Extended Horizons and the Family Firm". International Journal of the Economics of Business, 6(1), 41-55.

Jones, J. J. (1991). "Earnings Management During Import Relief Investigations". Jornal of Accounting Research, 29(2), 193-228.

Klein, A. (2002). "Audit committee, board of director characteristics, and earnings management". Journal of Accounting and Economics, 33, 375-400.

La Porta, R.; Lopez-de-silanes, F. y Shleifer, A. (1999). "Corporate Ownership Around the World". The Journal of Finance, LIV(2), 471-517.

La Porta, R.; Lopez-de-Silanes, F.; Shleifer, A. y Vishny, R. (2000). "Investor protection and corporate governance". Journal of Financial Economics, 58, 3-27.

Maury, B. (2006). "Family ownership and firm performance: empirical evidence from Western European corporations". Journal of Corporate Finance, 12, 321-341.

Morck, R.; Shleifer, A. y Vishny, R. W. (1988). "Management ownership and market valuation - An empirical analysis". Journal of Financial Economics, 20, 293-315.

Mustakallio, M.; Autio, E. y Zahra, S. A. (2002). "Relational and contractual governance in family firms: Effects on strategic decision making". Family Business Review, 15(3), 205-222.

Ohlson, J. (2014). "Accruals: An Overview". China Journal of Accounting Research, 7(2), 65-80. 
Osma, B. G.; Gill, B.; Noguer, D. A. y Clemente, A. G. (2005). "La investigación sobre Earnings Managements". Revista Española de Financiación y Contabilidad, 34(127), 1001-1033.

Sánchez, R. Í. y Fuentes, F. P. (2008). "Persistencia del resultado contable y sus componentes: implicaciones de la medida de ajustes por devengo". Revista Española de Financiación y Contabilidad, 37(137), 32-61.

Shleifer, A. y Vishny, R. (1997). "A Survey of Corporate Governance". The Journal of Finance, LII(2), 737-783.

Shleifer, A. y Wolfenzon, D. (2002). "Investor protection and equity markets". Journal of Financial Economics, 66, 3-27.

Wang, D. (2006). "Founding Family Ownership and Earnings Quality". Journal of Accouting Research, 44(3), 619-656.

Warfieid, T. D.; Wild, J. y Wild, K. (1995). "Managerial ownership, accounting choices, and informativeness of earnings". Journal of Accounting and Economics, 20, $61-91$.

Zellweger, T.; Kellermanns, F.; Chrisman, J. y Chua, J. (2011). "Family Control and Family Firm Valuation by Family CEOs: The Importance of Intentions for Transgenerational Control". Organization Science, 23(3), 1-18. 